\title{
EI sesgo de equiprobabilidad como dificultad para comprender la incertidumbre en futuros docentes argentinos.
}

\author{
José Ma Cardeñoso, Universidad de Cádiz (España) \\ Amable Moreno, Universidad Tecnológica Nacional de Mendoza (Argentina) \\ Esther García-González, Universidad de Cádiz (España) \\ Rocío Jiménez-Fontana, Universidad de Cádiz (España)
}

Recibido el 12 de Octubre de 2016; aceptado el 16 de Enero de 2017

\section{El sesgo de equiprobabilidad como dificultad para comprender la incertidumbre en futuros docentes argentinos.}

\section{Resumen}

En este trabajo se describe el uso del sesgo de equiprobabilidad, identificado en el razonamiento de 908 estudiantes para profesor de secundaria de Matemática y de Biología, de la provincia de Mendoza, Argentina. Analizamos la utilización de argumentos justificativos de las respuestas dadas a doce preguntas de investigación en las que se les pide a los estudiantes justificar la asignación de un cierto grado de la estimación de la probabilidad a un determinado fenómeno que se le plantean. La elección de los fenómenos se realizó teniendo en cuenta el contexto en el que se presentaba y el grado de probabilidad; se realiza convenientemente para que los items usados sean representativos de los contextos de juego, el contexto cotidiano y el contexto físico-natural.

Palabras clave. Tendencias de pensamiento probabilístico, sesgos probabilísticos, equiprobabilidad, formación competencial docente.

A equiprobabilidade como dificuldade em compreender argentinos incerteza nos futuros professores.

\section{Resumo}

Neste artigo o uso de viés equiprobabilidade identificados no raciocínio de 908 alunos para o professor do ensino médio de Matemática e Biologia, na província de Mendoza, Argentina é descrito. Analisamos o uso de justificação para as respostas às doze perguntas de investigação que são feitas para os alunos para justificar a atribuição de um certo grau de estimativa de probabilidade de um fenómeno e eventos que são apresentados. A escolha de situações foi seleccionado considerando o teor do produto e do grau de probabilidade; Realiza-se convenientemente para os itens utilizados são representativos dos contextos do jogo, cotidiano e o contexto físico-natural.

Para citar: Cardeñoso, J.M., Moreno, A., García-González, E \& Jiménez-Fontana, R. (2017). El sesgo de equiprobabilidad como dificultad para comprender la incertidumbre en futuros docentes argentinos. Avances de Investigación en Educación Matemática, 11, 145 - 166. 
Palavras chave. Tendências de pensamento probabilístico, bias probabilística, equiprobabilidade, a formação de competências de professores. teachers.

The equiprobability bias as a difficulty to understand uncertainty in prospective Argentine

\section{Summary}

This paper describes the use of the equiprobability bias, which has been identified in the reasoning of 908 prospective secondary school teachers of Mathematics and Biology, in the province of Mendoza, Argentina. We analyse the arguments justifying the responses given to twelve research questions in which students are asked to justify the assignment of a certain degree of probability for a given phenomenon and the events that arise. The situations were selected taking into account the content of the item and the degree of probability so that the items used are representative of the context of games, daily life and physical-natural context. training.

Keywords. Probabilistic thinking trends, probabilistic bias, equiprobability, teacher competency

L’équiprobabilité comme difficulté à comprendre l'incertitude dans les futurs enseignants Argentins.

\section{Résumé}

Dans cet article, l'utilisation de équiprobabilité biais identifié dans le raisonnement de 908 étudiants pour enseignant de mathématiques et de biologie, dans la province de Mendoza, en Argentine est décrite. Nous analysons l'utilisation de la justification des réponses aux questions de douze questions de recherche qui sont posées aux élèves pour justifier l'attribution d'un certain degré d'estimation de la probabilité d'un phénomène et les événements qui sont présentés. Les situations ont été choisi compte tenu du contenu de l'article et le degré de probabilité; est idéalement effectuée pour les éléments utilisés sont représentatifs des contextes du jeu, le contexte de tous les jours et le contexte physique naturel.

Mots-clés. Tendances de la pensée probabiliste, polarisation probabiliste, équiprobabilité, la formation des compétences des enseignants

\section{Motivación e Introducción}

A partir la reflexión y análisis sobre los resultados de las diferentes investigaciones realizadas en torno a las ideas probabilísticas de los docentes en el contexto español, nuestro interés actual se centra en la mejora de la implementación del currículo formativo de los futuros profesores en relación el campo estocástico, en el contexto argentino. Para ello necesitamos aproximarnos a las ideas que estos estudiantes tienen sobre las nociones básicas del pensamiento probabilístico. Pues, como nos indican Vásquez \& Alsina (2015), nuestros estudiantes van a ser los responsables de la docencia de la estadística y la probabilidad en la educación obligatoria. Es, por tanto, un requerimiento imprescindible, para obtener una formación adecuada en nuestros egresados, empezar por conocer sus ideas y concepciones probabilísticas; información necesaria para poder aproximarnos al reto de diseñar e implementar procesos formativos apropiados que les facilite claves para asumir la responsabilidad, como futuros docentes, que la sociedad les demanda.

En este artículo nos centramos en analizar como nuestros estudiantes, futuros docentes de formación secundaria, entienden el mundo indeterminista, primer peldaño 
sobre el que sustentar la comprensión de la incertidumbre en la que está inmersa nuestra sociedad actual. Para ello es necesario tener desarrollada una cierta concepción del mundo que integre la imprevisibilidad de sus interacciones, cuestión que pensamos es afrontable en nuestras disciplinas. Es decir, a través de la comprensión de la incertidumbre, intentamos promover en nuestros estudiantes una nueva visión más compleja de la cotidianeidad, cuestión para la que se requiere construir una conceptualización adecuada de la naturaleza indeterminista de los fenómenos en general y de los educativos en particular.

En este sentido presentamos y analizamos los resultados de una investigación recientemente desarrollada (Moreno, 2014), que se ha focalizado sobre una de las temáticas que siguen candentes en el campo de la Educación Matemática en las últimas décadas y ya tratada, bien desde el campo de la psicología o bien desde la educación estocástica, como es el uso de razonamientos sobre la base de los heurísticos (Tversky \& Kahneman, 1974). Presentaremos para ello los datos obtenidos en la investigación citada, uno de cuyos objetivos principales fue la caracterización de los tipos de respuesta, a preguntas sobre la asignación de probabilidad a determinados sucesos y su argumentación, dadas por los participantes en la investigación, futuros docentes de Educación Secundaria.

La finalidad global de dicha investigación fue la tipificación de sistemas de ideas, que hemos denominado Tendencias de Pensamiento Probabilístico (definidas inicialmente en Azcárate, 1996). El trabajo de Azcárate (1996), realizado con un pequeña muestra de futuros profesores de Primaria, dio lugar a unos potenciales interrogantes que se confirmaron, a través de una nueva investigación, con una muestra representativa de la población de profesores de Educación Primaria en Andalucía (Cardeñoso, 2001). La línea de investigación se continúa quince años más tarde con la investigación base de este artículo (Moreno, 2014), donde se analizan dichas tendencias entre la población de estudiantes en los Institutos de Formación del Profesorado de Secundaria, en Argentina.

Tomando como referencia la perspectiva de los modelos mentales propuesta por Johnson-Laird (1983), entendemos que las personas captan el mundo exterior a través de las representaciones mentales que construyen del mismo. Cuando un modelo mental ha sido útil para un sujeto en varias ocasiones, es posible que todo él, o algunas de sus partes sean guardadas en la memoria de largo plazo, acrecentando así el bagaje de conocimiento del sujeto. Así, Nercessian (1992) considera a los modelos mentales como niveles de análisis intermedios entre el fenómeno y el modelo final resultante. Siguiendo esta teoría, las cosmovisiones que tienen los sujetos al respecto de la probabilidad, y que utilizan como saber de referencia para interpretar las situaciones afectadas por incertidumbre, se pueden considerar como un modelo mental o tendencia de pensamiento desde el que analizar la realidad (Moreno \& Cardeñoso, 2014).

Conocer estas tendencias entre los docentes es decisivo para lograr alcanzar una alfabetización estocástica básica en los ciudadanos del siglo XXI (Gal, 2005), formación que se reclama desde múltiples instancias como instrumento fundamental para intervenir adecuadamente en el entorno en que estamos inmersos. Consideramos que en las etapas educativas de carácter obligatorio y postobligatorio, el docente no solo ha de educar el razonamiento probabilístico de los futuros ciudadanos sobre situaciones 
inciertas per se, sino que, siguiendo la lógica de una metodología de trabajo por proyectos, han de centrarse en el estudio de problemáticas socio-naturales que superan la reflexión matemática de los estudiantes, abriendo la problemática que los origina a temáticas que interfieren con cuestiones de carácter mucho más cotidiano.

En consecuencia, nuestro interés como formadores también se dirige hacia la educación científica que los docentes han de procurar en la población estudiantil. En el caso de la educación primaria, los docentes afrontan la responsabilidad de impartir tanto matemáticas como ciencias. Sin embargo, en la educación secundaria, la docencia de estas dos áreas recae en diferentes profesionales. Considerando el mandato curricular, el objeto de enseñanza del campo indeterminista, formalmente hablando, le corresponde a los docentes de matemáticas, aunque se ha de admitir que para la comprensión y elaboración de significados conceptuales del currículo de ciencias, la comprensión de la incertidumbre es fundamental; por ejemplo, hay múltiples nociones en el ámbito de las ciencias biológicas que tienen un fuerte componente aleatorio ${ }^{1}$.

Es por tanto objeto de nuestro interés analizar como los futuros docentes de secundaria, tanto de matemáticas como de biología, comprenden la incertidumbre como un aspecto fundamental que han de atender para el desarrollo del pensamiento estocástico en la educación obligatoria y postobligatoria, vital para comprender el mundo actual (Moreno, 2014).

\section{Marco de Referencia}

Para afrontar la investigación nos replanteamos el sentido y alcance de la utilización del razonamiento heurístico (Tversky \& Kahneman, 1974) desde revisiones posteriores aportadas por Kahneman (2002), con la teoría del proceso dual. Chernoff (2012) analiza ampliamente el heurístico de representatividad, en base a la idea de que, cuando las personas se enfrentan a una pregunta difícil, y tal vez tienen pocos recursos (por ejemplo, tiempo y herramientas) para analizar la pregunta, su respuesta rápida consistirá a menudo en sustituir la difícil cuestión por otra, cuya respuesta es producto de un proceso más fácil, que se conoce como atributo de sustitución. Esta idea, según entendemos, se compagina con el sentido de adjetivo de la palabra heurística, que como afirma Kahneman (2002, p.466), se puede utilizar en dos sentidos: "el sustantivo se refiere al proceso cognitivo, y el adjetivo de atributo heurístico especifica la sustitución que se produce en un juicio particular". Entendemos que esta sustitución puede explicar en parte el sentido del uso del sesgo de equiprobabilidad.

En investigaciones citadas, tanto en la formación de docentes de Primaria como de Secundaria, hemos encontrado diversos indicadores de existencia de razonamiento basado en creencias implícitas entre los diferentes participantes. Las respuestas a diversas tareas donde se les solicitaba su estimación de probabilidad nos permitieron realizar ciertas reflexiones que nos llevaron a la confirmación del uso de razonamientos

\footnotetext{
1 No podemos olvidar que gran parte de las nociones escolares que se desarrollan en el currículo correspondiente a áreas como la física y química y la propia matemática, están más ligadas al pensamiento determinista, donde lo seguro supera ampliamente a la incertidumbre, que se intenta comprender en los resultados del área estocástica
} 
heurísticos y el uso de diferentes sesgos descritos ampliamente en la literatura correspondiente, asociados al campo estocástico.

Para el artículo que nos ocupa, los datos aportados provienen, como ya hemos indicado, de una amplia muestra de futuros docentes de secundaria (Moreno, 2014). Focalizamos la presentación en una de las tipologías de respuesta que dan los futuros docentes, que son aquellas asociadas al sesgo de equiprobabilidad, evidenciado en los experimentos de Lecoutre (1992) y que describe la creencia de los sujetos en la equiposibilidad de todos los sucesos asociados a cualquier experimento aleatorio o fenómeno incierto. Incluso se encuentra, como indican Serrano, Batanero, Ortiz y Cañizares (1998, p. 8), "en aquellas situaciones en que no es aplicable el principio de indiferencia o donde no hay una simetría física", donde, sin embargo los sujetos afirman que los resultados al ser un fenómeno no determinista depende del azar, argumentación fundamental en la que reconocemos la tendencia de pensamiento hacia la incertidumbre (Cardeñoso, 2001). En el sesgo de equiprobabilidad los sujetos consideran que dicha impredicibilidad es inherente a la aleatoriedad del fenómeno y en consecuencia, puede ocurrir o no ocurrir, ignorando cualquier otro análisis sobre la equidistribución y simetría del aleatorizador y tampoco el análisis combinatorio de los posibles resultados esperables. En consecuencia, se disocia el uso del razonamiento combinatorio de las situaciones en que interviene el azar, perdiendo los sujetos la oportunidad de cuestionarse el diferente peso de los distintos elementos que constituyen el espacio muestral.

Desde las ideas expuestas, analizamos los razonamientos de los sujetos, justificativos del grado de probabilidad asignado, que estén apoyados en el argumento de equiprobabilidad. Para ello podemos tomar como referencia alguna de las dos formas que entendemos como posible interpretación del sentido de su utilización:

a) Bien desde la teoría de la dualidad de Kahneman (2002), mediante el heurístico de sustitución que se produce en un juicio particular, sustituyendo el problema por otro más sencillo de analizar, que permite al sujeto definir la equiprobabilidad del evento planteado;

b) Bien desde la creencia del sujeto en la distribución justa (uniforme) que pudiera explicar el alto y persistente uso del sesgo de equiprobabilidad (Gauvrit \& Morsanyi, 2014).

Las investigaciones previas confirman el elevado uso de estos tipos de argumentos, que configuran un sesgo del razonamiento probabilístico de los sujetos. Consideramos, como Batanero, Chernoff, Engel, Lee y Sánchez (2016), que la asunción de la equiprobabilidad de los resultados es subjetiva (salvo en los casos en que la equidad impregna los juegos de azar, como fenómenos asociados a la modelización laplaciana o clásica), y mantenemos que esta visión impide la aplicación de la probabilidad a una amplia variedad de fenómenos físico-naturales o cotidianos donde dicha presunción no puede ser válida.

En definitiva, este tipo de argumentación apoyada en la equiprobabilidad es muy atrayente y fácil de aceptar como explicación de la incertidumbre de ocurrencia de los sucesos, pero que ocasiona problemas en determinados contextos, como nos indican Batanero y Serrano (1995, p.18), 
La idea de equiprobabilidad es muy atrayente para definir la aleatoriedad, como se ve, por ejemplo, al definir las muestras aleatorias -todos los elementos tienen la misma probabilidad de ser elegidos-... cuando desplazamos la aplicación de la idea de probabilidad a situaciones del mundo físico-natural, nos encontramos con que no podemos aplicar el principio de equiprobabilidad.

\section{Metodología}

Dada la finalidad de la investigación, que es caracterizar las ideas de una muestra significativa de docentes, seleccionamos una metodología analítico-descriptiva y aplicamos el método de encuesta. Para ello utilizamos un cuestionario con 24 ítems que versaban sobre diferentes aspectos implicados en el pensamiento probabilístico, de los cuáles en este trabajo solo se analizan 12, los relativos a la estimación de la probabilidad. En dichos ítems, los participantes debían elegir entre una valoración baja, media o alta, de ocurrencia del suceso que se les planteaba y justificar su respuesta.

\subsection{Participantes}

Este cuestionario fue completado por 908 estudiantes para profesor de secundaria en matemática y biología, de la provincia de Mendoza en Argentina. Participaron 583 estudiantes del título de Profesorado de Matemática para la Educación Secundaria, correspondientes a los nueve institutos de formación docente de la provincia de Mendoza, distribuidos en los cuatro niveles que integran la carrera. Con respecto a los estudiantes del Profesorado de Biología para la Educación Secundaria, se contó con la participación de 325 de los cinco institutos de formación docente de la misma provincia y distribuidos también en los cuatro niveles.

\subsection{Instrumento}

Como se ha indicado, se aplicó un cuestionario, que diseñó y aplicó previamente Cardeñoso (2001), sobre el cual se realizaron algunas modificaciones para adaptarlo al contexto socio cultural y a las características propias del lenguaje.

El proceso de construcción del cuestionario, realizado por Cardeñoso (2001), partió de tipificar las respuestas obtenidas en el estudio de Azcárate (1996) y fue sometido a un proceso de validación del contenido, externo (validación de expertos) e interno (estudio piloto). A dichos expertos se les solicitó la valoración, tanto de los enunciados, como del contenido de los ítems, su pertinencia y adecuación a la finalidad de la investigación, mediante un formulario que cumplimentaron y que conllevó la construcción final del cuestionario. El estudio piloto posterior permitió matizar los enunciados y la presentación de los diferentes ítems para una mayor comprensión de los participantes. En la investigación de Moreno (2014) se realizó un nuevo estudio piloto dirigido, como hemos indicado, fundamentalmente a reformular los enunciados de cara a una mejor adaptación a las formas de expresión del castellano en Argentina y facilitar su compresión por los nuevos participantes.

Como ya hemos indicado, en cada uno de los 12 ítems considerados en este artículo, el estudiante debe realizar una primera estimación probabilística de los sucesos presentados, en una escala ordinal de tres categorías: baja, media y alta; y a continuación debe argumentar su decisión, bien eligiendo alguna de las cuatro 
argumentaciones cerradas que se les propone, bien mediante sus propias argumentaciones en la opción abierta que les oferta.

Entre las opciones cerradas, las argumentaciones respondían a diferentes concepciones de la probabilidad y en todos los casos se presentaba una relacionada con la idea de equiprobabilidad. En el presente escrito centramos el análisis en los participantes que seleccionaron dicha opción, para justificar su asignación de probabilidad en los diferentes ítems. Como por ejemplo en el ítem 4, se enunciaban las siguientes opciones cerradas:

Item 4. La confianza que tengo en que me toque algún regalo en una rifa, en la que participo con alguno de los 10.000 números vendidos para el viaje de estudios del colegio, es: Baja__ Media___Alta

1. Porque tengo muchos más números en contra que a favor de que me toque un premio.

2. Porque así lo indica la proporción entre los números que compré con relación al total de los vendidos.

3. Porque siempre puede tocar o no tocarme, siempre tengo un $50 \%$ de las posibilidades.

4. Porque nunca me ha tocado nada, ni a ninguna persona que conozca, en todos los sorteos en los que he participado.

5. Porque.

En la misma línea se presentaban los 12 ítems que se indican a continuación y que en el cuestionario completo, de 24 ítems, iban intercalados con los otros 12 ítems que evaluaban otros contenidos. En su presentación y análisis conservamos la numeración original que tenían en el cuestionario cumplimentado por los participantes (Tabla 1).

\section{Tabla 1. Ítems del cuestionario relativos a la estimación de la probabilidad}

\begin{tabular}{|c|c|}
\hline Ítem & Enunciado \\
\hline 1 & $\begin{array}{l}\text { Durante una tarde jugamos a lanzar dos dados legales y acordamos que gana quien } \\
\text { acierta el resultado de sumar los números obtenidos. La confianza que tengo en ganar } \\
\text { eligiendo el } 7 \text { para toda una tarde de juego, es... }\end{array}$ \\
\hline 4 & $\begin{array}{l}\text { La confianza que tengo en que me toque algún regalo en una rifa, en la que participo con } \\
\text { alguno de los } 10.000 \text { números vendidos para el viaje de estudios del colegio, es... }\end{array}$ \\
\hline 6 & La confianza que tengo en que amanezca un día frío el 14 de Octubre, es... \\
\hline 9 & anza que tengo en conocer una persona famosa el mes que viene, es... \\
\hline 10 & $\begin{array}{l}\text { En una mesa de juego se dispone de una caja de fichas, que contiene } 29 \text { fichas negras y } \\
16 \text { amarillas. La confianza que tengo en que salga una ficha negra, a lo largo de toda una } \\
\text { tarde de juego, es... }\end{array}$ \\
\hline 11 & $\begin{array}{l}\text { La confianza que tengo en sacar una bola roja de una urna que contiene } 5 \text { bolas blancas, } \\
5 \text { rojas y } 1 \text { azul, es... }\end{array}$ \\
\hline 12 & $\begin{array}{l}\text { La confianza que tengo en que se produzca un deterioro del medio ambiente de mi } \\
\text { localidad, el próximo año, es... }\end{array}$ \\
\hline 16 & $\begin{array}{l}\text { La confianza que tengo en conseguir las horas de cátedra de } 8^{\circ} \text {, el primero de la Escuela } \\
\text { secundaria de mi barrio cuando me reciba, es... }\end{array}$ \\
\hline 17 & vient \\
\hline 18 & $\begin{array}{l}\text { fianza que tengo en que me encuentre con un atasco, el sábado antes de Navidad, } \\
\text { centro de la ciudad, es... }\end{array}$ \\
\hline 19 & $\begin{array}{l}\text { La confianza que tengo en que, en un edificio de seis vecinos, en el primer intento } \\
\text { consiga pulsar el timbre del portero automático que corresponde a la puerta de un amigo } \\
\text { sin saber dónde vive, es... }\end{array}$ \\
\hline 22 & La confianza que tengo en que el próximo sismo que ocurra en Mendoza, sea un sismo \\
\hline
\end{tabular}


destructivo es...

El cuestionario se aplicó, como ya hemos indicado, en los trece institutos de formación docente a la muestra descrita. Fue pasado por uno de los autores en cada uno de los institutos mencionados, distribuidos en distintos puntos de la provincia de Mendoza. Fue completado individualmente sin interacciones y los estudiantes contaron con todo el tiempo que necesitaron para completarlo y no manifestaron falta de comprensión de los enunciados.

En relación al grado de probabilidad que se puede considerar más adecuado en cada suceso, este fue asignado según la propuesta realizada por los 18 expertos consultados (formadores en el campo de MIDE, Psicología y Educación Matemática), que sugerían seguir el siguiente criterio: estimación baja (B) si su probabilidad es inferior al 0,50; media (M), si la probabilidad de que ocurra el suceso es aproximadamente de un 0,50; y alta (A) si su probabilidad es superior al 0,50. Por otro lado, los ítems están caracterizados, según los fenómenos de referencia, en tres contextos denominados, de Juego cuando sean formulados sobre situaciones relativas al mismo, Cotidianeidad del resolutor, cuando se representan situaciones cercanas al entorno cotidiano, y cuando las situaciones eran de carácter más científico, las agrupamos en el contexto FísicoNatural.

En la Tabla 2 se clasifican los ítems que analizamos en el trabajo según el contexto y según la estimación probabilística realizada por los expertos. Vemos que la presencia de los ítems correspondientes nivel de probabilidad medio representan al 17\% (2 ítems) de los mismos. En consecuencia, si los participantes no presentasen el sesgo de la equiprobabilidad, la frecuencia de uso de dicho argumento debiera ser mínima en las justificaciones de la estimación de probabilidad demandada; sobre ello trataremos en el siguiente apartado.

Tabla 2 Clasificación de los items del cuestionario por contexto, en función del nivel de probabilidad estimado por los expertos

\begin{tabular}{clllc}
\hline \multirow{2}{*}{ Nivel de confianza } & \multicolumn{4}{c}{ Contexto (ítems asociados) } \\
\cline { 2 - 5 } & \multicolumn{1}{c}{ Juego } & \multicolumn{1}{c}{ Cotidiano } & \multicolumn{1}{c}{ Físico-Natural } & Total \\
\hline Bajo & 2 (ítems 1, 4) & 3 (ítems 9, 16, 19) & 2 (ítems 17, 22) & 7 \\
Medio & 1 (ítem 11) & 0 & 1 (ítem 6) & 2 \\
Alto & $1($ ítem 12) & 1 (ítem 10) & 1 (ítem 18) & 3 \\
\hline Total & 4 & 4 & 4 & 12 \\
\hline
\end{tabular}

Las respuestas de los estudiantes fueron analizadas mediante técnicas estadísticas descriptivas y multivariantes; encontrándose cuatro tendencias de pensamiento probabilístico (Moreno, 2014), similares a las encontradas en los estudios de Azcárate (1996) y Cardeñoso (2001), con algunas matizaciones. Las argumentaciones que dieron los estudiantes en la opción abierta del cuestionario fueron analizadas y categorizadas utilizando las informaciones referidas al estudio de heurísticos y sesgos. 


\section{Análisis}

Desgranamos a continuación nuestros resultados empíricos obtenidos en las respuestas al cuestionario referidas al sesgo de equiprobabilidad. En el análisis de las respuestas del cuestionario aplicamos diversas técnicas estadísticas, como tablas de frecuencias, y sus gráficos correspondientes y el test Chi cuadrado.

\subsection{Análisis de los ítems de estimación probabilística argumentados desde la equiprobabilidad}

La distribución de la variable "número de sucesos argumentados desde la equiprobabilidad en la estimación probabilística realizadas por los estudiantes", se presenta en la Figura 1. En ella se puede apreciar la inflación en el uso de la argumentación de equiprobabilidad en la mayoría de los sujetos, ya que se esperaba dicha argumentación tan sólo en dos (11 y 6) de los 12 ítems y tanto la moda como la mediana del número de argumentos de equiprobabilidad corresponden a cuatro ítems, siendo la media muy próxima a este valor.

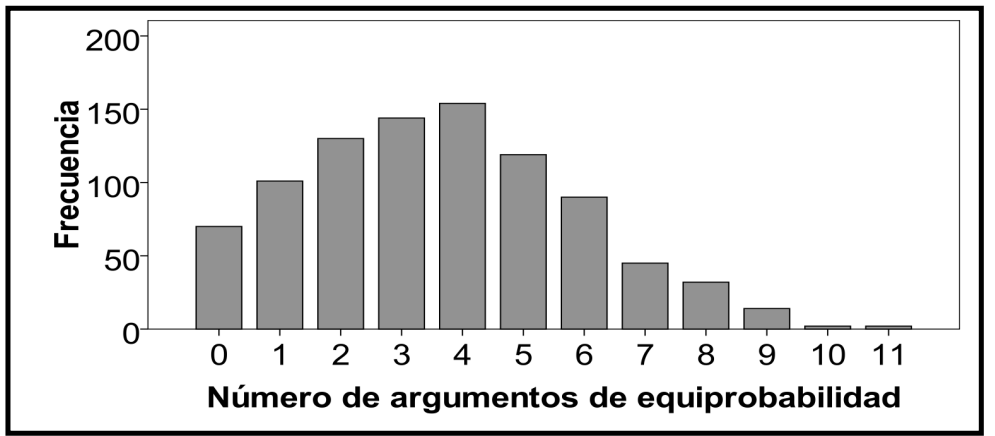

Figura 1. Distribución del número de sucesos argumentados desde la equiprobabilidad por los participantes

El análisis de dicha variable, en el conjunto de los 908 estudiantes de Matemática y de Biología de la provincia de Mendoza, nos permitió obtener los valores de los estadísticos que se muestran en la Tabla 3. Dichos valores indican que la variable en estudio presenta una importante variabilidad, dada por su desviación estándar $(2,23)$ y su coeficiente de variación porcentual $(61,29 \%)$.

Tabla 3 Estadísticos descriptivos de la frecuencia de sucesos argumentados desde la equiprobabilidad

\begin{tabular}{|c|c|}
\hline Estadístico & Valor del estadístico \\
\hline Valor mínimo & 0 \\
\hline Valor máximo & 11 \\
\hline Media aritmética & 3,64 \\
\hline Mediana & 4 \\
\hline Desviación estándar & 2,23 \\
\hline Coeficiente de variación porcentual & $61,29 \%$ \\
\hline
\end{tabular}

Si se analiza la variable en cada uno de los grupos de profesorados (Figura 2), podemos comprobar que existen diferencias significativas entre los resultados en los 
estudiantes de Matemática en relación con los de Biología. Es decir que, de manera global se puede deducir que el uso de la equiprobabilidad es significativamente mayor entre los estudiantes de Biología que entre los estudiantes de Matemática, con un valor $p$ de 0,037 en el test Chi cuadrado de homogeneidad de las dos muestras. Pero si el análisis se realiza por contexto, únicamente en el contexto de Juego se presentan diferencias significativas entre los de Matemática y los de Biología (Moreno, 2014).

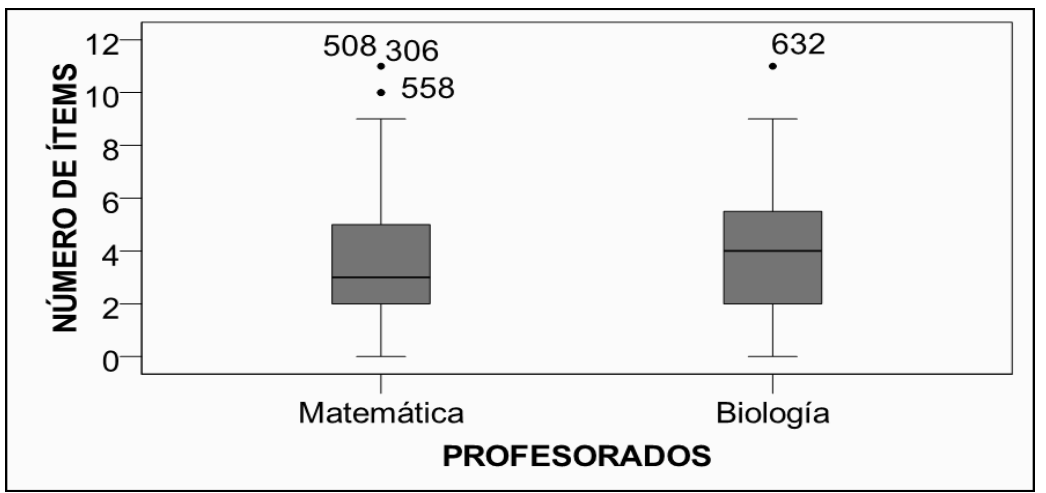

Figura 2. Distribución del número de sucesos argumentados desde la equiprobabilidad por los participantes por grupo

En el diagrama de cajas y bigotes de la Figura 2, se aprecia también la presencia de tres valores atípicos que argumenta la estimación probabilística de los sucesos desde la equiprobabilidad en diez u once de los doce items analizados, muy por encima de su grupo de referencia. Aunque no menos llamativo es el grupo de sujetos (cercano al 7 \%) con cero argumentaciones desde la equiprobabilidad que muestra la Figura 1.

\subsection{Argumentación de equiprobabilidad en sucesos no equiprobables}

Por otra parte, si se consideran únicamente los diez ítems que involucran sucesos no equiprobables, eliminando los resultados de los ítems 11 y 6 , y consideramos la frecuencia de sucesos argumentados desde la equiprobabilidad entre dichos ítems en toda la muestra, obtenemos la distribución presentada en la Figura 3. Es decir, que en el caso de sucesos no equiprobables, el $50 \%$ de los estudiantes asignan el argumento de equiprobabilidad a tres o más sucesos de los diez ítems planteados. Ello nos lleva a considerar la alta tasa del uso del sesgo de equiprobabilidad entre los sujetos de la muestra.

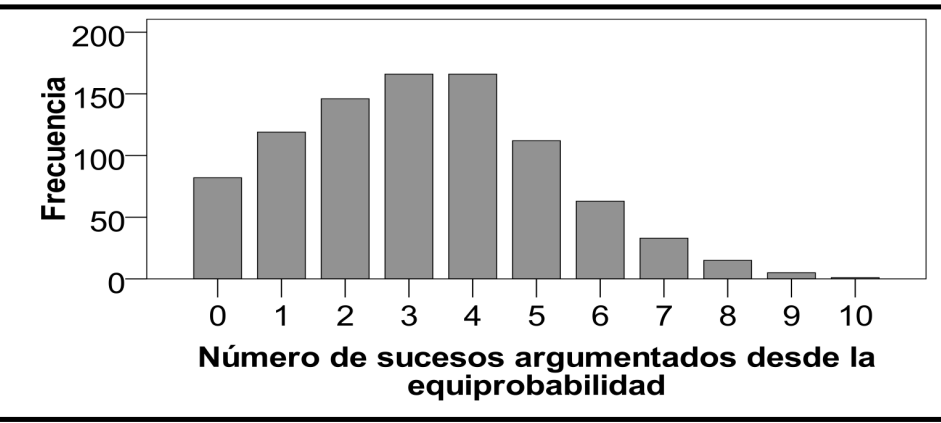

Figura 3. Distribución del número de sucesos no equiprobables argumentados desde la equiprobabilidad 
Como vemos en la Figura 3, hay que destacar la existencia de un grupo de sujetos cercano al 8\%, que nunca usan esta argumentación, representando el límite inferior de la desviación.

Tabla 4. Estadísticos descriptivos de la frecuencia de sucesos no equiprobables argumentados desde la equiprobabilidad

\begin{tabular}{lc}
\hline Estadístico & Valor del estadístico \\
\hline Media aritmética & 3,21 \\
Mediana & 3 \\
Desviación estándar & 2,0 \\
Coeficiente de variación porcentual & $62,34 \%$ \\
\hline
\end{tabular}

Los estadísticos referidos a estos diez ítems, quedan recogidos en la Tabla 4 que nos refleja de nuevo un uso muy alto de la argumentación de equiprobabilidad, para tratarse de sucesos no caracterizables como equiprobables.

\subsection{Análisis de argumentos de equiprobabilidad por contexto y especialidad del estudiante}

En la Tabla 5 hemos calculado el porcentaje de estudiantes que argumentan la estimación probabilística desde la equiprobabilidad en cada ítem en los diferentes contextos considerados. La tabla presenta los ítems ordenados según uso de la equiprobabilidad entre los estudiantes para profesorado de secundaria de Matemáticas, Biología y el global de la muestra.. En las últimas filas se presenta la media del porcentaje de argumentos desde la equiprobabilidad en ítems que comparten el mismo contexto.

Tabla 5. Porcentajes de estudiantes que argumentan la equiprobabilidad en cada item

\begin{tabular}{lccccc}
\hline Ítem & Contexto & $\begin{array}{c}\text { Probabilidad } \\
\text { estimada }\end{array}$ & Matemáticas & Biología & $\begin{array}{c}\text { Matemáticas. y } \\
\text { Biología }\end{array}$ \\
\hline 1 & Juego & $\mathrm{B}$ & 45,63 & 53,85 & 48,57 \\
4 & Juego & $\mathrm{B}$ & 15,27 & 28,31 & 19,93 \\
6 & Físico-Natural & $\mathrm{M}$ & 33,62 & 34,77 & 34,03 \\
9 & Cotidiano & $\mathrm{B}$ & 28,13 & 36,92 & 31,28 \\
10 & Juego & $\mathrm{A}$ & 11,66 & 19,38 & 14,43 \\
11 & Juego & $\mathrm{M}$ & 16,98 & 24,00 & 19,49 \\
12 & Físico-Natural & $\mathrm{A}$ & 36,02 & 31,69 & 34,47 \\
16 & Cotidiano & $\mathrm{B}$ & 40,14 & 39,08 & 39,76 \\
17 & Físico-Natural & $\mathrm{B}$ & 45,45 & 37,85 & 42,73 \\
18 & Cotidiano & $\mathrm{A}$ & 12,86 & 8,31 & 11,23 \\
19 & Cotidiano & $\mathrm{B}$ & 15,95 & 20,00 & 17,40 \\
22 & Físico-Natural & $\mathrm{B}$ & 50,77 & 50,15 & 50,55 \\
\hline Media Contexto Físico-Natural & & 41,46 & 38,62 & 40,44 \\
Media Contexto Cotidiano & & 24,27 & 26,08 & 24,92 \\
Media Contexto Juego & & 22,38 & 31,38 & 25,61 \\
\hline \multicolumn{2}{l}{ Promedio. Total } & & 29,37 & 32,03 & 30,32 \\
\hline
\end{tabular}

Estos resultados muestran que el uso de la equiprobabilidad, en promedio es menor en el contexto de Juego y en el contexto Cotidiano, en primer lugar por los estudiantes 
de Matemática, seguidos por los estudiantes de Biología. Mientras que en el contexto Físico-Natural, el uso es menor por los estudiantes de Biología y mayor por los estudiantes de Matemática. En la Tabla 6 se presentan los estadísticos descriptivos del porcentaje de estudiantes que aplican la equiprobabilidad por especialidad y globalmente. En promedio, los estudiantes de Biología son los que más usan la equiprobabilidad, en tanto los estudiantes de Matemática son los que presentan una menor argumentación de equiprobabilidad.

Tabla 6. Estadísticos descriptivos del porcentaje de estudiantes que argumentan desde la equiprobabilidad por item

\begin{tabular}{lccc}
\hline & Matemáticas & Biología & Matemáticas y Biología \\
\hline Mínimo & 11,66 & 8,31 & 11,23 \\
Primer Cuartil & 15,78 & 23,00 & 18,97 \\
Mediana & 30,88 & 33,23 & 32,66 \\
Tercer Cuartil & 41,47 & 38,16 & 40,50 \\
Media & 29,37 & 32,03 & 30,32 \\
Máximo & 50,77 & 53,85 & 50,55 \\
Desviación Estándar & 14,39 & 13,02 & 13,57 \\
\hline Coeficiente de variación & $49,00 \%$ & $40,66 \%$ & $44,76 \%$ \\
\hline
\end{tabular}

\subsection{Relación entre el nivel estimado de probabilidad por expertos y por estudiantes}

En este apartado se analiza la estimación probabilística que hacen los estudiantes argentinos. Al analizar la asociación entre la estimación media por ítem y la frecuencia de uso la argumentación de equiprobabilidad, en el conjunto de los participantes en el estudio, no pudimos rechazar la hipótesis de independencia (valor $p=0,241$ en el test Chi cuadrado), por lo que asumimos la independencia.

Si se compara la estimación probabilística que realizan los participantes en el estudio (Tabla 7) con la estimación realizada por los expertos, se puede calcular que el 48,15\% de los estudiantes del profesorado de Matemáticas asignan la misma estimación en los ítems con estimación baja por parte de los expertos, el 64,93\% cuando la estimación de los expertos es media y el 45,63\% si es alta. En el caso de los estudiantes de Biología, el 40,69\% asigna la misma estimación que los expertos a los sucesos con asignación probabilística baja, el 71,84\% a los sucesos con asignación probabilística media, y finalmente el $46,05 \%$ a los sucesos con asignación probabilística alta.

De manera global, podemos concluir que de los ítems de sucesos con asignación probabilística baja por los expertos, solo el 45,94\% de los participantes dan la misma estimación y de los sucesos con estimación probabilística alta, solamente el 45,78\% dan la misma estimación, por lo que más de la mitad de la muestra disiente de la estimación experta, cuando no se puede esperar una estimación media. La razón es que potencialmente utilizan el sesgo de equiprobabilidad que en su sentido heurístico o con el atributo de sustitución. 
Tabla 7. Porcentajes de estudiantes según niveles de estimación probabilística en cada item

\begin{tabular}{|c|c|c|c|c|c|c|c|}
\hline \multirow[t]{2}{*}{ Ítem } & \multirow[t]{2}{*}{$\begin{array}{c}\text { Estimación } \\
\text { Experta }\end{array}$} & \multicolumn{3}{|c|}{$\begin{array}{l}\text { Estimación probabilista del } \\
\text { estudiante (Matemáticas) }\end{array}$} & \multicolumn{3}{|c|}{$\begin{array}{l}\text { Estimación probabilística del } \\
\text { estudiante (Biología) }\end{array}$} \\
\hline & & Baja & Media & Alta & Baja & Media & Alta \\
\hline 1 & B & 27,79 & 54,03 & 16,30 & 18,15 & 68,31 & 12,00 \\
\hline 4 & B & 77,70 & 18,35 & 2,74 & 68,62 & 28,31 & 2,15 \\
\hline 6 & M & 46,14 & 48,89 & 3,95 & 35,08 & 61,54 & 2,46 \\
\hline 9 & B & 75,30 & 20,93 & 3,26 & 70,46 & 26,46 & 2,15 \\
\hline 10 & A & 3,26 & 41,85 & 54,37 & 4,62 & 44,92 & 50,15 \\
\hline 11 & $\mathrm{M}$ & 10,63 & 80,96 & 7,55 & 6,46 & 82,15 & 10,46 \\
\hline 12 & A & 32,42 & 52,14 & 14,41 & 25,85 & 51,69 & 20,62 \\
\hline 16 & B & 27,27 & 60,55 & 11,66 & 21,54 & 62,77 & 14,46 \\
\hline 17 & B & 24,19 & 59,52 & 15,78 & 22,77 & 65,54 & 14,77 \\
\hline 18 & A & 10,98 & 20,41 & 68,10 & 12,62 & 18,77 & 67,38 \\
\hline 19 & B & 65,87 & 29,33 & 3,43 & 50,38 & 36,00 & 3,38 \\
\hline \multirow[t]{2}{*}{22} & B & 38,94 & 51,80 & 8,75 & 32,92 & 59,69 & 7,08 \\
\hline & & 36,71 & 44.90 & 17.53 & 30,79 & 50,51 & 17.26 \\
\hline
\end{tabular}

\subsection{Aplicación de la equiprobabilidad en función de las tendencias de pensamiento probabilístico}

En esta sección analizamos el uso de la equiprobabilidad en estudiantes con diferentes tendencias de pensamiento probabilístico (Cardeñoso \& Azcárate, 2004). A partir de la aplicación del sistema de nueve categorías descrito en trabajos previos (Azcárate, 1996; Cardeñoso, 2001), mediante un análisis cluster, obtuvimos diferentes tendencias de pensamiento entre los participantes en nuestro estudio (Moreno, Cardeñoso, \& González-García, 2014) que sintéticamente son:

- Tendencia Determinista, que niega la existencia del azar, aunque con una buena capacidad para el cálculo que le permite tratar el azar en términos de probabilidades. Se afirma que el fenómeno no es aleatorio, porque cómo hay una regla de cálculo de probabilidades que nos permite saber su posible resultado, se aplican las matemáticas, que son consideradas deterministas. Cuando no puede responder a la evidencia de la incertidumbre, se califica el fenómeno de excepcional. Como para el determinismo el azar sólo puede ser epistemológico, se consideran preferibles las teorías científicas de las que se desprenden leyes en las que no tiene cabida el azar.

- La Tendencia Personalista, también de corte pre-indeterminista: en ella el azar y lo indeterminado son cuestiones de carácter mágico o fenomenológico, esotérico, cabalístico, del destino o de casualidades, como razón de los sucesos fortuitos; no se valora su posible cálculo.

- La Tendencia de Incertidumbre, que se limita a reconocer la existencia de fenómenos de azar y sucesos aleatorios y no les busca explicación. Utiliza las llamadas intuiciones primarias sobre los fenómenos y sucesos imprevisibles y son los que más reconocen los resultados como equiprobables.

- Tendencia Contingente, que caracteriza a los sujetos que discriminan los fenómenos aleatorios de lo determinista y supeditan lo real a lo posible. No sólo busca saber 
que ocurre, sino también conocer las causas que originan la variación de lo esperado respecto a lo posible.

En la Tabla 8 se muestra el uso del argumento de la equiprobabilidad en función de las tendencias que se caracterizaron en el conjunto de la muestra. Se puede observar un importe uso de la equiprobabilidad entre las tendencias de pensamiento hacia el determinismo y hacia la incertidumbre, tanto en el caso de futuros docentes de matemáticas como de Biología. Estos resultados coinciden en gran medida con los obtenidos por Cardeñoso (2001), en profesores de Primaria, quien informa de la gran resistencia de esta idea en el pensamiento probabilístico de los sujetos.

Tabla 8. Porcentajes de items argumentados desde la equiprobabilidad por tendencia de pensamiento y tipo de estudiante

\begin{tabular}{lcccc}
\hline & Incertidumbre & Contingencia & Personalismo & Determinismo \\
\hline Matemática & 46,80 & 15,92 & 18,58 & 34,67 \\
Biología & 44,50 & 15,25 & 8,33 & 44,17 \\
\hline
\end{tabular}

Contrastando los datos con los obtenidos en el estudio que ha servido de referente, en general, el uso del sesgo de equiprobabilidad fue inferior en el caso de los profesores en activo de Primaria españoles (Cardeñoso \& Azcárate, 2004) que en el caso de los estudiantes para profesor de secundaria argentinos, tanto de Biología, como de Matemáticas.

\subsection{Argumentos de equiprobabilidad}

Como hemos indicado, el instrumento está compuesto por ítems de opción múltiple, con cuatro opciones de respuesta cerradas y una abierta. En los 12 ítems considerados en este trabajo, la mayor parte de los sujetos se decantan por alguna de las opciones de respuesta cerrada ya tipificada, para justificar su asignación de estimación de la probabilidad de ocurrencia del fenómeno; estas ya han sido analizadas en los apartados previos en relación al uso de la equiprobabilidad. Pese a ello, en la respuesta abierta, hemos logrado encontrar variadas expresiones para argumentar la asignación de una confianza media a la probabilidad del suceso apoyadas de nuevo en la idea de equiprobabilidad (Moreno \& Cardeñoso, 2016). El mayor uso de las argumentaciones relacionadas con la equiprobabilidad entre los participantes, tanto de Matemática como de Biología se presenta en el ítem 1: Durante una tarde jugamos a lanzar dos dados legales y acordamos que gana quien acierta el resultado de sumar los números obtenidos. La confianza que tengo en ganar eligiendo el 7 para toda una tarde de juego es...

En esta situación algunos estudiantes del Profesorado de Matemática acusan la dificultad en la definición del espacio muestral y del suceso del que se debe estimar su probabilidad, bien por concepciones subjetivistas del azar:

S171: Tengo tres posibilidades sumando los puntos de los dados...

O se aprecia la creencia en la equiprobabilidad de resultados como cuando afirman:

S122: Porque hay las mismas posibilidades para cualquiera de los números... 
O si son entendidas desde el heurístico de sustitución y responden a una cuestión más sencilla que la que se le plantea, como se vislumbran en el siguiente ejemplo:

S317: Tengo tanta posibilidad de que salga o de que no salga depende de tu suerte...

Hemos encontrado argumentaciones similares desde la equiprobabilidad, en otros ítems donde las respuestas se basan en la indeterminación intrínseca del fenómeno, que se apoya en el atributo de sustitución al contestar a una cuestión más sencilla como es identificar como indeterminista la situación. En estos casos el término heurística tiene sentido adjetivo desde la teoría dual, y proporciona una respuesta no pertinente a la cuestión formulada.

Por ejemplo, podemos observar esta característica en los siguientes argumentos expuestos por estudiantes del Profesorado de Biología para justificar su asignación Media en el ítem 4: La confianza que tengo en que me toque algún regalo en una rifa, en la que participo con alguno de los 10.000 números vendidos para el viaje de estudios del colegio es...

S068: Es el azar puedo ganar o no.

S136: Porque puede que salga mi número o no.

S233: Creo que no depende de la cantidad de números vendidos o de lo que compre, creo que es exclusivamente azar.

S279: Porque el hecho que tenga comprado más de la mitad de los números no asegura el premio y puede que me toque.

Otras explicaciones semejantes se presentaron en el Ítem 17: La confianza que tengo en que corra viento mañana en mí localidad es...

S244: Porque puede o no correr viento.

$\mathrm{O}$ en el ítem 22: La confianza que tengo en que el próximo sismo que ocurra en Mendoza, sea un sismo destructivo es...

S206: Porque es igualmente posible que un sismo sea destructivo o que no lo sea.

En definitiva, podemos concluir que en el total de la muestra, contando con el uso de la opción de respuesta equiprobable, correspondiente a una de las opciones cerradas de cada ítem, más las respuestas abiertas clasificadas como de equiprobabilidad, se usa el argumento de equiprobabilidad con una alta frecuencia en la mayoría de los ítems. Argumento que no siempre era consistente con la estimación media de la probabilidad otorgada por la estimación experta de esta probabilidad en los ítems (Tabla 9).

Como podemos ver en la tabla, entre los ítems estimados de baja probablidad, tanto por expertos como por estudiantes, el $74,45 \%$ de los que dan una estimación baja usan, sin embargo la equiprobabilidad en el ítem 4 . El 73,57\% en el ítem 9 y el 63\% en el 19. Por otro lado, en los ítems con estimación de probabilidad alta tanto por expertos como por estudiantes, el $52,86 \%$ de los que estima una alta probabilidad sigue argumentando equiprobabilidad en el ítem 10 y el $67 \%$ en el 18.

Respecto a los estudiantes que dan una estimación media de probabilidad, se llega hasta un $60,24 \%$ de equiprobablidad en ítems con previsión experta baja, como en el caso del ítem 17; hasta un uso máximo $81,39 \%$ más de razonamiento equiprobable 
cuando se trata del ítem 11 con previsión experta como de estimación media y por último si es alta la previsión, como en el caso del ítem 12, se detecta hasta un 51,98\% de uso de dicho razonamiento.

Tabla 9. Porcentajes de items argumentados por la equiprobablidad desde cada cota de estimación global

\begin{tabular}{ccrrr}
\hline Ítem & $\begin{array}{c}\text { Estimación } \\
\text { Experta }\end{array}$ & \multicolumn{3}{c}{$\begin{array}{c}\text { Estimación probabilística del estudiante (Matemáticas y } \\
\text { Biología) }\end{array}$} \\
\cline { 3 - 5 } & & Baja & Media & Alta \\
\hline 1 & B & 24,34 & 59,14 & 14,76 \\
4 & $\mathrm{~B}$ & 74,45 & 21,92 & 2,75 \\
6 & $\mathrm{M}$ & 42,18 & 53,41 & 3,41 \\
9 & $\mathrm{~B}$ & 73,57 & 22,91 & 2,86 \\
10 & $\mathrm{~A}$ & 3,74 & 42,95 & 52,86 \\
11 & $\mathrm{M}$ & 9,14 & 81,39 & 8,59 \\
12 & $\mathrm{~A}$ & 30,07 & 51,98 & 16,63 \\
16 & $\mathrm{~B}$ & 25,22 & 61,34 & 12,66 \\
17 & $\mathrm{~B}$ & 23,68 & 60,24 & 15,42 \\
18 & $\mathrm{~A}$ & 11,56 & 19,82 & 67,84 \\
19 & $\mathrm{~B}$ & 63,55 & 31,72 & 3,41 \\
22 & $\mathrm{~B}$ & 36,76 & 54,63 & 8,15 \\
\hline Promedio & & 34,86 & 46,79 & 17,45 \\
\hline
\end{tabular}

\section{Conclusiones e Implicaciones}

De manera global podemos concluir que los participantes en el estudio hacen uso desmedido del argumento de equiprobabilidad para justificar su estimación probabilística; siendo significativamente mayor este argumento entre los estudiantes de Biología que los de Matemáticas. La dificultad se presenta mayoritariamente en los sucesos relativos al contexto Físico-Natural y al Cotidiano; mientras que en el contexto de Juego la dificultad aparece cuando la determinación del espacio muestral y del suceso al que se le debe asignar una estimación de la probabilidad, requiere de uso de nociones combinatorias, como es el caso del ítem 1.

Si bien únicamente, le corresponde una probabilidad media a dos sucesos del cuestionario, los estudiantes asignan en promedio la equiprobabilidad, aproximadamente a cuatro sucesos; el cincuenta por ciento de los estudiantes argumentan la estimación probabilística desde la equiprobabilidad en cuatro o más sucesos de los doce.

Por otra parte, menos de la mitad de los alumnos realizan una asignación probabilística consistente con la otorgada por los expertos como sucesos con asignación baja y alta. Si cruzamos los datos con las tendencias de pensamiento probabilístico caracterizadas en el estudio, el uso de la equiprobabilidad es mayor en el grupo de participantes asociados a la Tendencia al Determinismo y a la Incertidumbre, que son las que manifiestan mayor falta de concepciones probabilísticas formales.

Estas conclusiones revelan la necesidad de mejorar la formación probabilística de los futuros profesores de Matemática y de Biología para el desarrollo de una actividad 
docente que les permita llevar a cabo la alfabetización probabilística de los estudiantes de secundaria. Se entiende que si el formador parte de la detección de las ideas previas de sus estudiantes pueden considerar la idoneidad cognitiva de su docencia (Font \& Godino, 2010) y, en consecuencia, adquiere relevancia el conocimiento de los significados personales de los estudiantes respecto de la probabilidad, que conjuntamente con la consideración del criterio de idoneidad epistémica, puede permitir que construyan los significados institucionales pretendidos. Y, tras la identificación de las tendencias de razonamiento de cada grupo de sujetos de cada aula, el formador podrá ajustar su estrategia didáctica formativa.

En este sentido planteamos como hipótesis a constatar en futuras investigaciones, que la argumentación desde la equiprobabilidad, unas veces corresponde con el razonamiento que induce al sujeto a considerar que en todo fenómeno afectado por incertidumbre los sucesos son "por naturaleza" equiprobables y recoge la equiparaciónde equignorancia y equiposibilidad que Rouan y Pallascio (1994) plantean en su investigación. Otras veces no responde a la demanda de asignar la probabilidad esperada, sino a la pregunta de si ese evento es del campo indeterminista, cuestión que identifican con la incertidumbre intrínseca del fenómeno. Así en el caso del ítem 4, la respuesta desde la equiprobabilidad que se le ofrece es "porque siempre puede tocar o no tocarme,..." que corresponde a la respuesta sobre la incertidumbre de la situación y no sobre su estimación de ocurrencia.

Podemos compartir con Díaz (2003), que la instrucción en probabilidad no es suficiente para superar los errores que cometen los egresados ni ayuda a superar obstáculos, por lo que es necesaria una confrontación que permita reconocer las concepciones alternativas de sus razonamientos intuitivos. Ante cualquier intervención formativa, es necesaria la confrontación de sus razonamientos intuitivos con las nuevas ideas apoyadas en un razonamiento más acorde con el estudio adecuado del contexto, como estrategia imprescindible para promover el cuestionamiento de sus ideas (Shaughnessy, 1977).

Por otra parte, la tendencia de pensamiento enfocado a la Contingencia, tiene el menor y casi despreciable porcentaje de presencia de heurísticos y sesgos (Moreno \& Cardeñoso, 2014; 2015). A lo cual, nosotros sugerimos considerar el análisis sobre la tendencia de pensamiento contingente, por ser el que se caracteriza por una buena identificación de fenómenos inciertos y minimiza el uso del razonamiento heurístico y sesgos asociados. Ello nos permite sugerir la utilización didáctica de la idea de contingencia en el aula de formación de profesores, pues prácticamente los estudiantes que apoyan en ella su razonamiento no poseen el sesgo de equiprobabilidad, por lo que debiéramos considerar esta forma de razonar como un nivel estable e interesante para la superación de los sesgos.

Otra clara consecuencia, es la necesidad de investigar sobre los posibles tratamientos didácticos del ámbito de la incertidumbre, en particular buscar estrategias que permitan a los futuros docentes comprender la incertidumbre y su incidencia en las situaciones de nuestro contexto actual para poder tratarlas adecuadamente en su futura labor profesional. Como sostiene Prigogine (1996), siempre hemos navegado en un océano de incertidumbres a través de archipiélagos de certidumbres locales, pues la ciencia clásica de la modernidad se ha basado en una concepción de orden y estabilidad, 
mientras que los fenómenos complejos, como los presentes en el sistema de crisis global, registran fluctuaciones, inestabilidad, azar, caos; cuestiones que ha de afrontar un educador actual que pretende afrontar nuevos retos en el marco de la educación para el desarrollo sostenible.

Son estas las competencias del profesor que le permiten afrontar el futuro, en el que hay que pensar con la incertidumbre, pero no la incertidumbre absoluta, como recomienda Franklin (2014). Los profesores de la escuela secundaria deberían lograr la comprensión de las conexiones que se pueden establecer entre la estocástica y otras áreas de estudio de la escuela media (ciencias experimentales, ciencias naturales, ciencias sociales, etc...). En el mismo sentido se incide desde la UNECE (2013, p.14) en la necesidad que los educadores desarrollen competencias que vayan más allá de la educación en su propia disciplina y que les permita "estar dispuestos a tomar medidas incluso en situaciones de incertidumbre".

Todo lo anterior en aras a alcanzar un cierto nivel de desarrollo profesional que permita ejemplificar la puesta en práctica de nuevas formas de actuar que promuevan, no solo un conocimiento estocástico más operativo, sino una valoración de su utilidad en el análisis de la realidad en múltiples contextos. En esta línea existen ya muchas aportaciones, que afrontan autores como Barth, Fischer, Michelsen, Nemnich \& Rode (2012) y Vega-Quirós (2012). Con lo que se podría superar lo que Sánchez (2009, p. 74) en su análisis de los programas echa de menos, "situaciones para tratar más a fondo el sesgo de la equiprobabilidad con experiencias cuyo espacio muestral no se reduzca a un conjunto equiprobable de resultados".

Para terminar coincidimos con Jiménez-Fontana, García-González, Azcárate \& Navarrete (2015), en que el estudio en la procesos formativos de situaciones y fenómenos implicados en procesos de desarrollo sostenible en contexto de situaciones socio-naturales cotidianas, puede ser un escenario idóneo para mediante el desarrollo de proyectos cercanos a la realidad de los sujetos. Así se puede potenciar desde la formación estocástica y científica de los futuros docentes, la conceptualización de la probabilidad como saber imprescindible que permita aproximar a los sujetos a la comprensión del mundo y la búsqueda de otro mundo posible (Azcárate \& Cardeñoso, 2011).

\section{Agradecimientos}

Este artículo es parte de los resultados de EDINSOST, Proyecto I+D+i2015, EDU2015-65574-R (MINECO/FEDER), Subvencionado por el Ministerio de Economía y Competitividad.

\section{Referencias}

Azcárate, P. (1996). Estudio de las concepciones disciplinares de futuros profesores de primaria en torno a las nociones de aleatoriedad y probabilidad. Granada: Comares.

Azcárate, P., \& Cardeñoso, J.M. (2011). La enseñanza de la estadística a través de escenarios: implicación en el desarrollo profesional. Bolema, Boletim de Educação Matemática, 24(40), 789-810. 
Barth, M., Fischer, D., Michelsen, G., Nemnich, C., \& Rode, H. (2012). Tackling the knowledge-action gap in sustainable consumption: insights from a participatory school programme. Journal of Education for Sustainable Development, 6(2), 301-312.

Batanero, C., \& Serrano L. (1995). La aleatoriedad, sus significados e implicaciones educativas. UNO, 5, 15-28.

Batanero, C., Chernoff, E., Engel, J., Lee, H., \& Sánchez, E. (2016). Research on teaching and learning probability, ICME-13 topical surveys. Berlín: Springer. DOI 10.1007/978-3-31931625-3_1.

Cardeñoso, J.M. (2001). Las creencias y conocimientos de los profesores de primaria andaluces sobre la Matemática escolar. Modelización de conceptos sobre la Aleatoriedad y Probabilidad. Tesis Doctoral. Universidad de Cádiz.

Cardeñoso J.M., \& Azcárate, P. (2004). Las concepciones de los profesores de primaria ante el conocimiento probabilístico: implicaciones para su formación. Revista de Educación de la Universidad de Granada, 17, 11-35.

Chernoff, E.J. (2012). Recognizing revisitation of the representativeness heuristic: an analysis of answer key attributes. ZDM - The International Journal on Mathematics Education, 44(7), 941-952.

Díaz, C. (2003). Heurísticas y sesgos en el razonamiento probabilístico. Implicaciones para la enseñanza de la Estadística. Actas 27 Congreso Nacional de Estadística e Investigación Operativa. Lleida: S.P.U. Lleida.

Franklin, K. (2014). The statistical education of teachers: an American Statistical Association policy. En K. Makar, B. De Sousa, \& R. Gould (Eds.), Proceedings of the Ninth International Conference on Teaching Statistics. Voorburg, The Netherlands: International Statistical Institute. Recuperado de: http://icots.info/9/proceedings/pdfs/ICOTS9_1A2_FRANKLIN.pdf.

Font, V., \& Godino, J.D. (2010). Inicio a la investigación en la enseñanza de las matemáticas en secundaria y bachillerato. En C. Coll (Ed.), Matemáticas: Investigación, innovación y buenas prácticas. Barcelona: Graó.

Gal, I. (2005). Towards" probability literacy" for all citizens: Building blocks and instructional dilemmas. En G. Jones (Ed.), Exploring probability in school (pp. 39-63). New York: Springer.

Gauvrit, N., \& Morsanyi, K. (2014). The equiprobability bias from a mathematical and psychological perspective. Advances in Cognitive Psychology, 10(4), 119-130.

Jiménez-Fontana, R., García-González, E., Azcárate, P., \& Navarrete, A. (2015). Dimensión ética de la sostenibilidad curricular en el sistema de evaluación de las aulas universitarias. El caso de la enseñanza aprendizaje de las Ciencias. Revista Eureka sobre Enseñanza y Divulgación de las Ciencias 12(3), 536-549.

Johnson-Laird, P.N. (1983). Mental models. Cambridge, MA: Harvard University Press.

Kahneman, D. (2002). Maps of bounded rationality: A perspective on intuitive judgment and choice (Nobel Prize Lecture). En T. Frangsmyr (Ed.), Les Prix Nobel. Recuperado de: www.nobel.se/economics/laureates/2002/kahnemann-lecture.pdf

Lecoutre, M.P. (1992). Cognitive models and problem spaces in purely random situations. Educational Studies in Mathematics, 23, 557-568. 
Moreno, A. (2014). Un estudio comparativo de las tendencias de pensamiento probabilístico de los profesores de biología y de matemática en formación. Tesis Doctoral. Universidad de Granada.

Moreno, A., \& Cardeñoso, J.M. (2014). Overview of prospective mathematics teachers' probabilistic thinking. En K. Makar, B. De Sousa, \& R. Gould (Eds.), Proceedings of the Ninth International Conference on Teaching Statistics. Voorburg, The Netherlands: International Statistical Institute. Recuperado de: http://icots.info/9/proceedings/pdfs/ICOTS9_1A2_FRANKLIN.pdf.

Moreno, A., \& Cardeñoso, J.M. (2015). La contingencia: la tendencia mayoritaria de pensamiento probabilístico en futuros profesores de matemáticas en secundaria. Didáctica de la Estadística, Probabilidad y Combinatoria, 2, 355-362.

Moreno, A., \& Cardeñoso, J.M. (2016). Un instrumento para la explicitación de tendencias de pensamiento probabilístico. Premisa, 18(69), 16-28.

Moreno A., Cardeñoso J. M. \& González-García F. (2014). El pensamiento probabilístico de los profesores de biología en formación. Bolema, Boletim de Educação Matemática 28(50), $1415-1442$

Nercessian, N. (1992). Construction and instructing: the role of "abstract techniques" in creating and learning physics. En Duschl, R., \& Hamilton, R. (Eds.). Philosophy of science, cognitive psychology and educational theory and practice (pp. 49-68). New York: SUNY

Prigogine, I. (1996). El fin de las certidumbres. Santiago de Chile: Andrés Bello.

Rouan, O., \& Pallascio, R. (1994). Conceptions probabilistes d'éléves marocains du secondaire. Recherches en Didactique des Mathématiques, 14(3), 393-428.

Sánchez E. (2009). La probabilidad en el programa de estudio de matemáticas de la secundaria en México. Educación Matemática, 21(2), 39-77.

Serrano, L., Batanero, C., Ortiz, J.J., \& Cañizares M.J. (1998). Heurísticas y sesgos en el razonamiento probabilístico de los estudiantes de secundaria. Educación Matemática, $10(1), 7-25$.

Shaughnessy, J.M. (1977). Misconceptions of probability: An experiment with a small- group, activity-based, model building approach to introductory probability at the college level. Educational Studies in Mathematics, 8, 295-316.

Tversky, A., \& Kahneman, D. (1974). Judgement under uncertainty: Heuristics and biases. Science. $185,1124-1131$.

UNECE (2013). Empowering educators for a sustainable future. Strategy for education for sustainable development tools for policy and practice workshops on competences in education for sustainable development. Recuperado de: http://www.unece.org/index.php?id=35136\&L=0

Vásquez, C., \& Alsina, A. (2015). El conocimiento del profesorado para enseñar probabilidad: Un análisis global desde el modelo del conocimiento didáctico-matemático. Avances de Investigación en Educación Matemática, 7, 27-48.

Vega-Quirós, M. (2012). El aprendizaje estadístico en la educación secundaria obligatoria a través de una metodología por proyectos. Estudio de caso en un aula inclusiva. Tesis doctoral. Universidad de Granada. 


\section{Referencia a los autores}

José M $\mathrm{M}^{\mathrm{a}}$ Cardeñoso, Universidad de Cádiz (España); josemaria.cardenoso@uca.es

Amable Moreno, Universidad Tecnológica Nacional de Mendoza (Argentina); amable.moreno@frrm.utn.edu.ar

Esther García-González, Universidad de Cádiz (España); esther.garcia@uca.es

Rocío Jiménez-Fontana, Universidad de Cádiz (España); rocio.fontana@uca.es 


\section{The equiprobability bias as a difficulty to understand uncertainty in prospective Argentine teachers}

José $\mathrm{M}^{\mathrm{a}}$ Cardeñoso, University of Cádiz (Spain)

Amable Moreno, National Technological University of Mendoza (Argentina)

Esther García-González, University of Cádiz (Spain)

Rocío Jiménez-Fontana, University of Cadiz (Spain)

We analyse the presence of the equiprobability bias in the reasoning of prospective mathematics and biology teachers in Mendoza (Argentina). This reasoning was used to justify the assignment of probability to the occurrence of some events.In the theoretical framework, we reflect on different points of view about reasoning biases and heuristics. We analyse them in the framework of the duality theory and in the consideration of the attribution substitution. We discuss the role of the subjects' mental representations as the intermediate space between everyday conceptions and scientific models.

The data are collected through a questionnaire of twelve items, completed by a sample of 908 future teachers. Each item presents an event of an indeterminate situation and the prospective teachers have to estimate its probability and provide a justification. Each item presents four closed options corresponding to prototypical answers, including an equiprobability option and an open-ended option.

The situations were selected considering the content of the item and the degree of probability. The situations are representative of three contexts: games of chance, daily context and physical-natural context. We analyse the responses given by the participants and identify a greater use of the reasoning of equiprobabilidad than that was expected; the contexts and the students' reasoning tendencies where this use becomes more evident are also identified. We conclude with an analysis of the implications for the education of teachers. 